\title{
What is the role of HER2-specific antibody immunity in patients with HER2-positive early breast cancer receiving chemotherapy plus trastuzumab?
}

\author{
Joe Mehanna ${ }^{1}$, Fady GH. Haddad ${ }^{2}$, Roland Eid ${ }^{2}$, Matteo Lambertini ${ }^{3}$, Hampig Raphaël Kouriée \\ ${ }^{1}$ Faculty of Medicine, Saint-Joseph University, Beirut, Lebanon; ${ }^{2}$ Faculty of Medicine, Hematology-Oncology Department, Saint-Joseph University, \\ Beirut, Lebanon; ${ }^{3}$ Department of Medical Oncology and Breast Cancer Translational Research Laboratory, Institut Jules Bordet and Université \\ Libre de Bruxelles (U.L.B.), Brussels, Belgium \\ Correspondence to: Matteo Lambertini, MD. Institut Jules Bordet and Université Libre de Bruxelles (U.L.B.), Boulevard de Waterloo 121, 1000 - \\ Brussels, Belgium. Email: matteo.lambertini85@gmail.com. \\ Comment on: Norton N, Fox N, McCarl CA, et al. Generation of HER2-specific antibody immunity during trastuzumab adjuvant therapy associates \\ with reduced relapse in resected HER2 breast cancer. Breast Cancer Res 2018;20:52.
}

Submitted Sep 04, 2018. Accepted for publication Sep 11, 2018.

doi: $10.21037 /$ tcr.2018.09.07

View this article at: http://dx.doi.org/10.21037/tcr.2018.09.07

Breast cancer is the most common malignancy among women worldwide and the leading cause of cancer death in many countries (1). In around $20 \%$ to $25 \%$ of breast cancer cases, there is an overexpression of HER2 or an amplification of its gene (HER2-positive subtype); HER2 is a transmembrane receptor implicated in tumor replication, invasion and dissemination (2). HER2-positive breast cancer is characterized by a worse prognosis, aggressive disease, higher mitotic index, worse nuclear grade and lymph nodes positivity (3).

The anti-HER2 monoclonal antibody trastuzumab inhibits proliferation and induces death via intracellular or extracellular mechanisms by targeting HER2-positive cells and inducing receptor internalization and degradation (4). Trastuzumab was the first approved targeted therapy against HER2-positive breast cancer. In the early stage, results from several trials suggested improved survival outcomes when adjuvant trastuzumab was added to chemotherapy with 5 -year disease-free survival (DFS) and overall survival (OS) exceeding $80 \%$ and $90 \%$, respectively (5). More recently, pertuzumab has been also approved in the adjuvant setting. Pertuzumab is an anti-HER 2 antibody with complementary mechanism of action to that of trastuzumab (6); this monoclonal antibody acts by preventing the dimerization of HER2 with HER3 (7). Dual anti-HER2 blockade with trastuzumab plus pertuzumab has proved to be a successful strategy also in the adjuvant setting leading to a 3-year DFS rate of $94.1 \%(8)$.
Despite the survival advantage associated with the addition of trastuzumab to the treatment of early breast cancer, a not negligible proportion of patients develop disease relapse. With the recent approval of pertuzumab in the early setting and the relatively small added benefit associated with its use, the identification of the patients who are more likely to benefit from anti-HER2 targeted therapies represents an area of crucial importance to be further developed in the coming years. In fact, besides HER2 itself, biomarkers predicting patients who may truly benefit from anti-HER2 agents are still lacking (2). Therefore, other predictors of response to targeted therapies beyond HER2 testing need to be identified. HER2-specific antibodies may be promising biomarkers on this regard.

The investigators of the NCCTG N9831 trial had previously shown evidence on the important role of immunity in HER2-positive early breast cancer by both gene expression profile analyses (9) and the evaluation of tumors infiltrating lymphocytes (TIL) (10). In the recently published analysis by Norton and colleagues conducted within the NCCTG N9831 trial, the investigators showed that the serum levels of endogenous antibody immunity specifically developed against HER2 (i.e., HER2-specific antibodies) increased only with the use of adjuvant trastuzumab (given sequentially or concurrently to chemotherapy as per study design) and not following the 
administration of chemotherapy alone (11). In addition, patients who experienced elevation in the levels of posttreatment HER2-specific antibodies had improved DFS compared to those in whom antibody levels remained normal. The 5 -year DFS rate after adjuvant trastuzumab was $84 \%$ among patients with elevated antibodies, contrarily to $60 \%$ in patients with normal levels (11).

This analysis raises the important questions on how increased serum levels of HER2-specific antibodies can be associated with better DFS, and if their levels should be routinely monitored when treating breast cancer patients with trastuzumab. Moreover, it remains to be proven if HER2-specific antibodies should be considered as a predictive factor for treatment effect and if their monitoring can inform on its duration, maintenance or discontinuation.

Trastuzumab is probably the primary mediator of activating the adaptive immune system as suggested by the NCCTG N9831 trial results in which adjuvant chemotherapy alone did not generate HER2-specific antibodies, contrarily to the combined treatment of paclitaxel and trastuzumab that resulted in an adaptive immune response in the majority of patients (12). It can be hypothesized that the generation of antibodies could be mediated via antibody-dependent cell mediated cytotoxicity (ADCC), a process leading to apoptosis and release of antigens in the circulation, or through the activation of antigen-presenting cells. Those cells can process antibodyHER2 complex in the tumor microenvironment (13). Another study examined specific antibodies against the HER2 extracellular and intracellular domains in patients receiving chemotherapy plus trastuzumab (14). Clinical response to trastuzumab and chemotherapy may be due to several pathways involved in the adaptive immune response (15).

The levels of HER2-specific antibodies were shown to be elevated prior to treatment in patients with HER2positive breast cancer, relatively to healthy individuals (14). However, antibodies to HER2 extracellular domain were increased in the adjuvant setting as compared to the metastatic disease, while antibodies targeting the intracellular domain were lower in the adjuvant setting. Moreover, the induction of HER2-specific antibodies by trastuzumab was more pronounced in patients with normal (low) pre-existing antibody levels as compared to patients with elevated levels at baseline. Also survival analyses showed that the presence of high preexisting antibody immunity to HER2 was associated with poorer progressionfree survival and OS (14).

Considering the timing for monitoring the serum levels of HER2-specific antibodies, in the NCCTG N9831 trial, serum samples were taken between 19 months and 24 months post-treatment (11). However, in another study, serum samples were collected around 4 months after initiation of adjuvant therapy (14). The best timing for measuring this biomarker after treatment initiation needs to be further explored considering its potential clinical implications.

Taken together, the available but limited data on the topic including the recent analysis by Norton and colleagues suggest that HER2-specific antibodies represent emerging promising biomarkers to be further explored in the setting of HER2-positive early breast cancer during anti-HER2 targeted therapy. Besides being able to target the HER2 extracellular domain and thus improving the response to therapy, trastuzumab therapy appears to also target the intracellular domain and other tumor antigens. We hypothesized that patients more likely to beneficiate from anti-HER2 targeted treatments are those who have normal baseline levels of antibodies and then are capable of generating significant levels of HER2-specific antibodies following treatment initiation.

In conclusion, results from the study by Norton and colleagues are encouraging and indicates a possible role of measuring the levels of HER2-specific antibodies to predict treatment response in patients with HER2-positive early breast cancer receiving chemotherapy plus trastuzumab. However, no strong conclusions on the potential clinical implications of these findings can be made particularly considering the small sample size (50 pairs of pre- and posttreatment serum samples) of the analysis. Therefore, larger studies are needed in the field to confirm these findings and potentially allow the incorporation of the monitoring of HER2-specific antibodies into daily practice in order to better select the patients that would better respond to antiHER2 targeted therapy or those with intrinsic treatment resistance.

\section{Acknowledgments}

Matteo Lambertini acknowledges the support from the European Society for Medical Oncology (ESMO) for a Translational Research Fellowship at the Institut Jules Bordet in Brussels (Belgium).

\section{Footnotes}

Provenance and Peer Review: This article was commissioned 
by the editorial office, Translational Cancer Research. The article did not undergo external peer review.

Conflicts of Interest: Matteo Lambertini served as a consultant for Teva and received honoraria from Theramex outside the submitted work. The other authors have no conflicts of interest to declare.

Ethical Statement: The authors are accountable for all aspects of the work in ensuring that questions related to the accuracy or integrity of any part of the work are appropriately investigated and resolved.

Open Access Statement: This is an Open Access article distributed in accordance with the Creative Commons Attribution-NonCommercial-NoDerivs 4.0 International License (CC BY-NC-ND 4.0), which permits the noncommercial replication and distribution of the article with the strict proviso that no changes or edits are made and the original work is properly cited (including links to both the formal publication through the relevant DOI and the license). See: https://creativecommons.org/licenses/by-nc-nd/4.0/.

\section{References}

1. Senkus E, Kyriakides S, Ohno S, et al. Primary breast cancer: ESMO Clinical Practice Guidelines for diagnosis, treatment and follow-up. Ann Oncol 2015;26:v8-30.

2. Lambertini M, Pondé NF, Solinas C, et al. Adjuvant trastuzumab: a 10-year overview of its benefit. Expert Rev Anticancer Ther 2017;17:61-74.

3. Arpino G, Milano M, De Placido S. Features of aggressive breast cancer. Breast 2015;24:594-600.

4. Vu T, Claret FX. Trastuzumab: updated mechanisms of action and resistance in breast cancer. Front Oncol 2012;2:62.

5. Slamon D, Eiermann W, Robert N, et al. Adjuvant trastuzumab in HER2-positive breast cancer. N Engl J Med 2011;365:1273-83.

Cite this article as: Mehanna J, Haddad FG, Eid R, Lambertini M, Kourie HR. What is the role of HER2-specific antibody immunity in patients with HER2-positive early breast cancer receiving chemotherapy plus trastuzumab? Transl Cancer Res 2018;7(6):1354-1356. doi: 10.21037/tcr.2018.09.07
6. Baselga J, Swain SM. Novel anticancer targets: revisiting ERBB2 and discovering ERBB3. Nat Rev Cancer 2009;9:463-75.

7. Harbeck N, Beckmann MW, Rody A, et al. HER2 Dimerization Inhibitor Pertuzumab - Mode of Action and Clinical Data in Breast Cancer. Breast Care (Basel) 2013;8:49-55.

8. von Minckwitz G, Procter M, de Azambuja E, et al. Adjuvant Pertuzumab and Trastuzumab in Early HER2Positive Breast Cancer. N Engl J Med 2017;377:122-31.

9. Perez EA, Thompson EA, Ballman KV, et al. Genomic analysis reveals that immune function genes are strongly linked to clinical outcome in the North Central Cancer Treatment Group n9831 Adjuvant Trastuzumab Trial. J Clin Oncol 2015;33:701-8.

10. Perez EA, Ballman KV, Tenner KS, et al. Association of Stromal Tumor-Infiltrating Lymphocytes With Recurrence-Free Survival in the N9831 Adjuvant Trial in Patients With Early-Stage HER2-Positive Breast Cancer. JAMA Oncol 2016;2:56-64.

11. Norton N, Fox N, McCarl CA, et al. Generation of HER2-specific antibody immunity during trastuzumab adjuvant therapy associates with reduced relapse in resected HER2 breast cancer. Breast Cancer Res 2018;20:52.

12. Knutson KL, Perez EA, Ballman KV, et al. Generation of adaptive HER2-specific immunity in HER2 breast cancer patients by addition of trastuzumab to chemotherapy in the adjuvant setting: NCCTG (Alliance) study N9831. J Clin Oncol 2013;31:abstr 522.

13. Guilliams M, Bruhns P, Saeys Y, et al. The function of $\mathrm{Fc} \gamma$ receptors in dendritic cells and macrophages. Nat Rev Immunol 2014;14:94-108.

14. Knutson KL, Clynes R, Shreeder B, et al. Improved Survival of HER2 + Breast Cancer Patients Treated with Trastuzumab and Chemotherapy Is Associated with Host Antibody Immunity against the HER2 Intracellular Domain. Cancer Res 2016;76:3702-10.

15. Sliwkowski MX, Mellman I. Antibody therapeutics in cancer. Science 2013;341:1192-8. 